9.--The solubility and consequently superior penetrability of the bichlorid is probably productive of the mercurial characteristic effects which seem ont of proportion to the amount of metal in doses of this salt; but it is not to be denied that chemic or direct neurotic influences coöperate with the metal in the more active preparations, and thus possess features of their own.

10.- Experimental evidence is opposed to probable formation of any compound in the body, and supports the belief that decomposition invariably and almost instantaneously follows its ingestion with the precipitation of mercury as minutely divided globules, from any preparation of which it forms the base.

\section{PUERPERAL ECLAMPSIA AND ITS TREATMENT.}

Read before the Marion County (Ind.) Medical Society.

\section{BY J. T. MCSHANE, M.D.}

INDIANAPOLIS, IND.

In presenting this paper, with the reports of the cases which have come under the writer's observation, together with the treatment and results, it would be ingratitude not to mention the name of the late Dr. Ellerslie Wallace, of Jefferson Medical College, Philadelphia, who, after a large experience, taught with emphasis the value of venesection in puerperal eclampsia. His hand with which he emphasized his words, with a deformed index finger, the result of a dissecting wound, was not more apparent to me when he said relative to this subject "you are on a life saving mission; do your duty fearlessly, and bleed for effect, not for ounces of blood," than when at the bedside of my first patient with eclampsia. It occurred in 1871 in the case of Mrs. W., primigravida, aged 35 years. Labor was slow and a severe headache was complained of which did not yield to free administration of bromid of potassium. After four hours of moderately hard labor the patient complained of intense throbbing pain in the head, sudden fright and blindness, followed almost immediately by a violent convulsion. As soon as the convulsion subsided, preparations were made for venesection, but before it could be done a second convulsion came on. Without further delay the arm was constricted and a large stream of blood allowed to flow. A third convulsion eame on while the blood was flowing. After this conrulsion passed off the patient's face, which had been dark, swollen and distorted, became pale and placid, the pulse became weak and thready. The bandage was removed from the arm and the flow stopped. The os uteri was pretty well dilated and with the assistance of Dr. S. C. Dove, of Westfield. Ind., whose office was less than a mile away, the patient was delivered of twins, both of which were still. A good recovery followed.

Mrs. G., now of this city, had convulsions with two confinements, and also early in a subsequent pregnancy. Dr. Rodman, of Zionsville, now of Denver, saw her during her first attack. She had some fifteen convulsions before bleeding, none after, and two hours later she was delivered of a mascerated child. In her second attack Dr. N. G. Harold, of Carmel, Ind., saw her with me. She had had seven or eight convulsions before my arrival. She was large and plethoric and an unusual amount of blood was required to produce the desired effect. Labor advanced slowly and in about two hours she was again seized with a convul- sion. The vein in the opposite arm was opened after which there was no more trouble, and as soon as clilatation was sufficient to admit of the application of the forceps she was delivered of a still child. When six months advanced in a subsequent pregnancy this patient had violent throbbing headache, a slight convulsion, clouded memory and intellect, all of which were immediately relieved by venesection. This treatment was repeated twice for the same symptoms, with the same happy results, before her term expired, when she was delivered of a healthy child, having no return of convulsions.

Mrs. W., lived at Nora, Ind., now of this eity. She was delivered in her first confinement of a healthy child without unusual labor or other symptoms. Some two hours later she was seized with convulsions. which were terrific in violence and came in quick succession. She had eighteen or nineteen convulsions, some of which were observed by Dr T. M. Hinshaw, who now lives in Westfield, Ind. On my arrival the doctor and the husband considered the case hopeless, and, indeed, it looked as if their opinion was correct. The patient was profoundly comatose, face almost black, swollen and distorted, leaving no trace of its former likeness, pulse quick and wiry, temperature high. As soon as the convulsion passed off a vein was opened and a large stream of blood allowed to flow. The amount drawn, though large, was disregarded, but the effects were eagerly watched. The face blanched, the pulse became thready and soft; the bandage was loosened. No more convulsions followed. The patient returning to semi-consciousness the next day heard her baby cry and exclaimed, "Whose baby is that?" Her recovery was not retarded. Her memory was thoroughly obliterated for a period of two days before and four days after delivery.

Mrs. S., multipara; a patient of Dr. W. O. Harland, now of Chicago. This patient was neither anemic nor plethoric. Soon after delivery she was seized with violent convulsions. She had taken chloral and chloroform without effect, and had had six or eight convulsions before my arrival. After a moderate bleeding convulsions subsided for a time. After an interval of two or three hours' rest, convulsions returned. Her pulse was hard and quick and the temperature was high. Blood was again drawn but in larger quantities, leading Dr. Harland to think the danger point was reached. In a letter received from Dr. Harland a few days ago, in answer to inquiry concerning this and another case, he says "we stopped the convulsions in Mrs. S., but we had to bleed her nearly to death to do it." The danger was more apparent than real. She had no more convulsions and was up and well in the usual time. A subsequent pregnancy was attended with a throbbing headache, disturbed vision, congested face and clouded memory, at the sixth month. A vein was opened and while the stream still flowed the grateful patient exclaimed "Oh, what a relief!" These symptoms reappeared at the seventh and eighth months, and the patient sought relief by the lancet both times. The delivery of a healthy child at term was normal.

Miss P., primigravida, also a patient of Dr. W. O. Harland. Convulsions came on early in her labor. She was freely bled after she had had five or six convulsions. The intervals were lengthened, but an occasional convulsion came on until the os was sufficiently dilated to admit the application of forceps. She was 
delivered of a still child, after which she had no more convulsions. She made a good recovery.

Miss H., primigravida. Large, strong, domestic; walked four miles to the house of an acquaintance. She was dazed and unable to give an account of her visit or from whence she came. In a short time she was seized with a convulsion. My arrival was delayed some hours on account of the messenger bearing no intelligence regarding the nature of the case. On my arrival five hours after the first convulsion she was comatose: her tongue was lacerated and bleeding. The family in whose house she was had no knowledge of her labor nor her pregnant state. An examination revealed both, the os being considerably dilated. Her convulsions had been frequent and severe, according to her great muscular development. After venesection she had no more convulsions. She shrank from her pains, and being unconscious the assistance of two strong men of the neighborhood was required to hold her in position in the bed. In about two hours, dilatation was sufficient to admit of the application of forceps, when she was delivered of a large still child. The return of consciousness was deferred thirty hours after delivery.

Mrs. D., multipara, was attended in her labor by Dr. Leavens. She complained of blinding, throbbing headache during the progress of her labor. This was lightly regarded, the physician thinking it would subside after delivery. Labor terminated at 4 o'clock P.M. At 2 oclock the following morning a summons reached me. She was in a convulsion when the messenger started, and was in a stupid state on my arrival. $A$ vein was opened and about a pint of blood taken when, the patient expressing a feeling of relief, the bandage was removed. Thirty minutes later she was attacked by a second convulsion. The vein in the opposite arm was opened and the blood allowed to flow until the face became pallid. The patient being large and plethoric a large quantity of blood was required. No further trouble followed and the patient's recovery was not retarded by the loss of blood.

Mrs. K., of Mattsville, Ind., was seized with a convulsion during her third confinement. The premonitory symptoms were pronounced and a vein was opened just as a convulsion came on. A healthy child was delivered an hour later and her recovery was uneventful.

Mrs. S., primigravida, was attacked with convulsions while under the care of Dr. K. C. Hershey, who then lived at Mattsville, Ind. The convulsions were hard and came in rapid succession. She had a large but uncounted number before my arrival. Her pains had been constant. severe and distressing, but not of an expulsive character. Free bleeding resulted in temporary relief. Examination revealed dilatation to the size of a silver half dollar, and the os presenting high up against the sacrum, the result of obliquity of the uterus. Gentle pressure against the fundus and slight traction with the finger brought the os down to its proper position, when the labor pains became strong and normal, with intervals of rest. Consciousness returned so that the patient conversed intelligently. After waiting three hours the patient was left in the care of Dr. Hershey, the attending physician. After some time had elapsed he was called away from the bedside. The history of the case shows that the pains lost their distinct character, becoming constant and distressing. After four hours intermission the convulsions reappeared. On my arrival in answer to a second summons the patient was in a moribund condition and soon expired. At the request of the husband and with the assistance of Dr. Hershey, the child was removed by Crsarean section. It was a perfect child, but as we expected, after so many convulsions, it was still.

Mrs. C., large, fleshy patient in fourth confinement. Had complained of throbbing headache for two days. A hurried messenger came with the report that she had fallen on the floor and they thought she was dying. Her term had expired and an examination revealed a dilated os, although no uterine pains had been complained of. She was in her fourth convulsion on my arrival. Her mouth was bleeding from laceration of the tongue. A vein was opened and a stream allowed to flow until the dark congested condition of her face gave place to pallor. Two hours later a still child was delivered and she made a good ecovery.

Mrs. S., now living in Kansas; during her first confinement was seized with a terrific convulsion. This was in all respects like a puerperal convulsion, but the patient having occasionally had epileptic symptoms, this was at first thought probably of that character. Chloral hydrate and bromid of potassium were freely given but in less than an hour a second convulsion came on which was followed by stupor and continued congestion of the face. My conclusion was that, although a slight epileptiform manifestation had been observed once or twice a year, this was puerperal, and fearing death of the child and danger to the life of the mother if the convulsions were repeated, she was bled until the desired effects were produced. No more trouble followed and she was soon delivered of a healthy child. It is a matter of regret that examinations of the urine were not made in each case reported. In the four cases in which analyses were made, there were traces of albumin in two. Those who have had the misfortune to witness cases of puerperal eclampsia will readily appreciate the delay which would attend such examination before treatment. The patient and child are in great danger and the necessity for prompt action engages the physician's time and attention. Whether there is or is not albuminuria, although of scientific interest, is at the instant of little importance. The treatment which relieves in the one case also relieves in the other.

The etiology of puerperal eclampsia is not definitely settled. Perhaps Löehlein covers the ground by stating that "no explanation of eclampsia has been fully established, and the disease has certainly more than one cause." Albuminuria is present in a majority of the cases, but this is not sufficient to afford a positive decision that the condition of the kidneys which produces albuminuria is the cause of eclampsia. Albuminuria is said to be present in five cases of pregnancy to one of eclampsia. It is pretty generally conceded that, whether the case is one of eclampsia gravidarum, parturientium or puerperarum, the arterial pressure is high, and this is true whether the blood is rich in red corpuscles or not. This condition may depend upon the action of the vasomotor system, or to increased volume of the circulatory medium, or both. The kidneys become clogged by congestion resulting from high tension, and their function is partially suspended, in which case albuminuria may or may not be present. Women in pregnancy are much inclined to accumulate flesh and when constructive metabolism is in excess of destructive meta- 
bolism, toxemia and plethora are simultaneously present. When albuminuria is present, unless chronic, the patient recovers without delay after cessation of convulsions, when the pressure is removed allowing the kidneys to assume their normal state. As a rule, the condition of the patient who is to become an eclamptic is not incompatible with comfortable health; the foudroyant out-break being the first announcement of departure from the physiologic state.

Reflex uterine irritation is said to be a cause of eclampsia in some cases. The fatal case of Mrs. $\mathbf{S}$. reported above, is probably one of this class. Obliquity of the uterus disturbed the normal expulsive character of the pains. When the uterus was righted the constant grinding, distressing pains ceased, giving place to normal labor. When left without support obliquity again took place, with disturbance of normal labor pains, and reappearance of convulsions. While in this case the obliquity seemed to be a disturbing factor, it is well known that such obliquity not infrequently exists without such disturbance. It would seem to be an interesting and curious fact that mal-presentations are very rarely attended with convulsions. The cases reported in this paper were all vertex presentations with the face to the right or left sacro-iliac synchondrosis. Dr. King asserts that convulsions scarcely ever occur in cross or shoulder presentations. A Dublin obstetrician says (Dublin Joumal Medical Sciences) that there is little fear of eclampsia if the child does not present by the head. This refers to eclampsia during labor; certainly not to cases occurring months before. There are exceptions to this rule. In a series of fifty-nine cases reported by Ramsbotham there were four breech presentations.

The annual of the Universal Medical Sciences for 1895 , records the researches of Rapin and Monier, who examined the blood of four women with eclampsia, and in three of the cases found a special bacillus, which they were able to cultivate, and when inoculated into animals caused symptoms of eclampsia.

Chamberland has experimentally proved that some poisonous element is present in the blood of eclamptic women, but the exact nature of the poison, or its origin, have not been determined. A rabbit is killed by the injection into its tissues of healthy human serum, when injected in the proportion of ten grams of the serum to every kilogram that the rabbit weighs. Chamberland has experimented extensively with the blood of eclamptic women, and has found the amount of serum of such blood necessary to kill a rabbit is less than the serum from a healthy person. In some cases one-half, and in others less than one-third the proportionate amount is sufficient to prove fatal.

It is believed by some investigators that the liver may have something to do with some of the cases of puerperal eclampsia. The healthy liver modifies certain poisonous elements brought to it from the intestines through the portal vein, and impairment of the liver which would wholly or in part, destroy or suspend this function would admit to the blood substances freighted with 'toxic. properties. Hahn, Masser and others have shown by experiments, by which the blood from the portal vein is emptied directly into the circulation, thereby depriving it of passage through the liver, nervous symptoms similar to those of eclampsia are produced. This is especially pronounced when the experiment is made after the inges- tion of meat. Their deduction is that these symptoms are due to the presence of carbamic acid in the blood, a substance which in passage through the liver is converted into urea. Further experiments show that when carbamic acid is introduced into the circulation not only the nervous symptoms are developed but the urine becomes scanty and albuminous.

Women in their first pregnancies, and also those who have more than one child in utero are most susceptible to eclampsia. Of the fourteen cases reported in this paper six were in cases of primipara, one of which had twins. In reference to the frequency of convulsions statistics are variable. The total number of deaths from eclampsia reported to the Board of Health in New York city in the nine years ending in 1875 , were 408 . The estimated maximum number of deliveries during that period was 284,000 , or nearly one death in 700 confinements.

The vital statisties furnished by the Indiana State Board of Health show the number of children delivered during twelve years and nine months to be 452,429. Deducting one birth from each case of doubtlets and two from each case of triplets the approximate number of labors represented in these reports is 449,000 . The number of deaths reported from eclampsia during this period is 440 or one in 1,020 deliveries. One series of 100,935 deliveries in the Philadelphia Board of Health reports, shows 94 deaths from eclampsia, or one in 1,073 confinements. Of women attacked Hecker found 1 in 522; Wieger 1 in 519; Kleinwächler and Galabin 1 in 500; Korman 1 in 600; Corson 1 in 300; Cazeaux 1 in 200 deliveries. It is estimated by some authors that the average is $1 \mathrm{in} 500$ labors. The health reports show the deaths only, but if this average is correct about 50 per cent. of the patients die. Parvin places the percentage of women attacked at 1 in 250 or 1 in $300 \mathrm{in}$ this country, and if this is correct the death rate from this cause as compared to the number of patients affected is reduced to about 28 to 30 per cent. Dr. Kemper, of Muncie, had nine cases of eclampsia in his one thousand labors reported. Eight of the women recovered and one died. After reference to the great fatality which attended eclampsia in the seventeenth and eighteenth centuries, Ramsbotham says in his work on obstetrics, "few cases now terminate unfortunately; and the favorable results are to be attributed to the extent to which bleeding and other evacuant means are carried.'

Patients who are bled in this disease do not suffer from anemia on account of the depletion, but regain their normal strength and color, and as a rule, are out of bed as early as though nothing unusual had transpired. Prof. Fordyce Barker insisted upon the unmistakable clinical evidence favorable to the employment of the lancet, and pleaded for its restoration in the management of puerperal eclampsia. Lusk says the claims of bleeding in eclampsia rest upon a substantial foundation, and that its special advantage lies in its prompt action.

Speigleberg says, "No means so quickly lessens arterial pressure, none so quickly restores their function to the kidneys, irritable from blood pressure, and few act so readily upon the excited vasomotor nerves, causing relaxation."

Patients with eclampsia are never able to sit while the operation is made, consequently a larger quantity of blood is required to produce the desired results than if the sitting posture could be assumed. It is 
much better not to bleed at all than to bleed ineffi- beneficial. Chloroform has been recommended and ciently. While the loss of a small amount of blood given in full anesthetic doses for the purpose of comwill do no harm it can do no good, and therefore it pletely paralyzing the motor centers. There is much brings probably the very best life-saving remedy in danger from prolonged anesthesia carried to the sureclampsia into disfavor and disrepute.

The life of the child in eclampsia parturientium is endangered by each succeeding attack, rarely persisting after the fourth or fifth convulsion. In proportion to its relative prompt action the life of the child and also that of the mother are enhanced by venesection: In claiming for the lancet a clean, safe, prompt, efficient and ever at hand remedy, the value of other remedies is not ignored. Dr. L. J. Hammond, Secretary of the Staff of Maternity Hospital, of Philadelphia, has kindly furnished me a history of nine cases which occurred in that institution during the last five years. All but one occurred in primigravida, and three women died. The treatment relied upon is depletion by purgation; chloral and bromids per rectum, veratrum viride, hot packs and anesthetics during the seizure, oleum tiglii is the cathartic relied upon. Two of the cases which recovered were bled, one to the amount of twenty-six ounces.

In answer to a letter addressed to the Philadelphia Lying-in Charity and Nurse School, Drs. W. Reynolds Wilson and Della M. S. Walker kindly prepared for me a table of the nine cases which occurred in that institution during the last four years. Previous history was negative in all the cases but two, one of which had vomiting of pregnancy, and the other had previous dropsy. These two patients died, the other seven recovered. One of the patients that recovered had sixteen, one had ten, one had three, and three had two convulsions. One of the patients who died had one, and in the other the word "frequent" takes the place, in the column, of the figure designating the number. The patient who had sixteen convulsions was bled. The others were treated by chloral, morphia and calomel. One exclusively by purgation, except that chloroform was used in all for the paroxysms. The average number of convulsions in these cases is small, owing doubtless, to the fact that the patients were in the hospital under the immediate attention of the physicians who were equipped with everything needful, thus securing prompt relief before the patients became so profoundly influenced. Whether the poison contained in the blood of an eclamptic acts directly upon the cortical cells of the brain is not demonstrable. It is a reasonable position to assume that it does, and that this poison, as well as reflex irritation from abnormally painful uterine contractions, disturb the cell power to control energy. The result is sudden liberation of energy manifested in a convulsive seizure.

Chloroform, chloral and morphia lessen reflex irritability and paralyze cell energy, thereby aiding in holding convulsions in abeyance; while diaphoresis, purgation and venesection relieve arterial tension and eliminate the poison.

The high arterial pressure which is almost universally present in eclampsia has lead many practitioners to believe that this disease does not occur in patients who do not bear depletion. Morphia, chloral and chloroform have been used with excellent results in a large number of cases. These agents may be used with advantage after depletion, or in the early history of an attack. After a patient has had a large number of convulsions and is in a state of coma, or near it, the administration of such drugs is not likely to prove gical degree, and added to coma, or tending coma, resulting from convulsions, it is capable of doing irreparable harm. The same may be said of large doses of morphia and chloral.

Dr. W. Reynolds Wilson, in his article on "The Use of Morphia in Eclampsia, with a Report of Two Cases," published in "The Annals of Gynecology and Pediatry," June 1892, a reprint of which the author has kindly furnished me, discusses this subject pretty thoroughly. In the second case reported by Dr. Wilson, morphia was given hypodermically in doses ranging from $\frac{1}{8}$ to $\frac{1}{2}$ grain. The history of the case shows the drug had a partially controlling influence, but "the patient was bled on account of vascular tension, shown in the congestion of the face, and throbbing of the arteries." Fifteen ounces were abstracted after which the patient had no more convulsions, but the morphia was continued and served a good purpose in controlling the restlessness.

Veratrum viride has been successfully employed in some cases reported. In large doses it slows the pulse, and as expressed by Hare, it "bleeds the patient into his own vessels" by filling up the mesenteric veins and thereby relieving arterial pressure. Dr. Fearn, of Brooklyn, in his article published in the American Journchl of Obstetrics on "Veratrum Viride in Large Doses as a Substitute for Blood-Letting in Puerperal Eclampsia" recommended the tincture of veratrum viride in doses ranging from fifteen minims to a teaspoonful repeated every five or ten minutes until the pulse became soft or vomiting occurred. After the arrest of convulsions he recommended the continuation of the drug for some hours in smaller doses and at longer intervals keeping the pulse below fifty per minute. In each case reported in this paper a very large dose of some active cathartic was given. Nitrite of amyl was used by Dr. W. F. Jenks, of Philadelphia, on the suggestion of S. Weir Mitchell. Ether had been used without avail. The inhalation of a few drops of nitrite of amyl immediately arrested the convulsions, but the patient had a dangerous uterine hemorrhage, which was attributed by Dr. Jenks to the action of the drug. The opinion of Dr. Jenks is corroborated by the experience of Dr. A. W. Brayton, of this city, who met with a dangerous uterine hemorrhage following the administration of one-fiftieth grain of nitro-glycerin, the action of which is similar to that of nitrite of amyl. The drug was given as a cardiac stimulant in a case of palpitation of the heart in a woman who had been delivered six days before.

Delivery should be faciliated in cases of eclampsia parturientium, when labor is difficult, and where the os uteri is dilated, by the application of forceps, or podalic version. Dilation may be hurried by the use of the colpurynter. A method has been proposed and practiced by Dührssen of Berlin which in his hands seems to have proved highly satisfactory. When the cervical canal is opened up, and the obstacle to delivery is the undilated os externum he makes four incisions reaching from the margin of the os up to the utero-vaginal junction. This he claims leaves no further obstacles to the delivery of the child, which can readily be effected without any fear of tearing the soft tissues. Thirty-five cases were treated by him, by this method, according to his published report, with- 
out the loss of a mother, and with a loss of only two children. The technique of this operation is explicitly stated in his report.

There is great danger to the life of the unborn child in cases of eclampsia. Its death not unfrequently occurrs during the first convulsion, often during the second, and after a number of convulsions the child is rarely born alive. The child is profoundly influenced and if its life is spared until birth it may die in convulsions soon after, as was the case with two of the cases reported by Drs. Wilson and Walker, of Philadelphia, and a case reported by Spence which was removed alive by the Cresarean section, the mother having died from eclampsia before there was any disposition to labor. Respiration is suspended during the early part of an eclamptic seizure, and is very imperfect until the attack passes entirely off, thus adding to the already bad condition of the maternal blood, rendering it inadequate to act as an oxidizing and purifying agent for the infantile circulation through the placenta. It is therefore fair to say Dührssen's cases must have been selected and operated upon immediately after the onset of the convulsions, otherwise more than two still births would have occurred in thirty-five labors. This is a possible illustration of the effect which overenthusiasm has on statistics.

\section{TRAUMATIC CARIES OF CERVICAL VERTEBR丑.}

A CASE WITH REPORT OF THE POST-MORTEM. BY L. G. NORTH, M.D. TECUMSEH, MICH.

Mr. R. J., aged 34 years, unmarried, an Englishman by birth and a resident of this country for fourteen years. By occupation a farm laborer and ditcher. In build very muscular, and rather thick set. He had great endurance, taking pride in the amount of work he could do in a day. His habits were all good, and when questioned in regard to syphilis asserted positively to the end that he had never known a woman.

About the middle of December 1894 while at work in a pig-pen he suddenly slipped and fell violently striking his right infra-mammary region upon the top of a low door, and falling from the door to the floor. He was aware that he bumped his head, but the injury to the side was the only one that he thought of any account.

From the time of the accident he was aware that he was not right, having pain in the right mammary and infra-manmary regions with constant shortness of breath. For this he consulted a physician and took medicine and used local applications for about two months, without decided improvement. About the middle of February 1895 while in the post office in a country store he was suddenly taken with a violent "catch" or "crick" in the back of the neck. He was able to get into a chair and from there on to a couch or settee, from which place he was not able to be moved for some five or six hours. From that time to the time of his death he was not able to turn his head or bend his neck without severe pain, and in fact did not move his head at all without supporting it with both hands. A favorite "hold," when it was necessary to move was with the left hand behind the occiput and the thumb of the right hand under the inner end of the supra-orbital ridge of the right eye. When not moving he often lay for a long time in this posi- tion. Following the severe attack above mentioned he continued to have pain in the right mammary and infra-mammary regions with soreness on motion, pressure and respiration, and before coming under observation of the writer he had two more similar attacks. The writer subsequently elicited the facts that the attacks occurred suddenly when the patient was somewhat improved and on assuming a vertical position. It was at first asserted that the second attack occurred in the night when the patient was in bed, but close questioning elicited the fact that he had risen to attend to a call of nature. During and between these attacks he had fever and acceleration of pulse but to what degree the writer was not able to learm. Soon after the first attack he began to cough and expectorate freely a muco-purulent material. The attending physician had counsel, but a definite diagnosis was not reached. The case came into the hands of the writer May 5, 1895. The patient was found lying on the right side with the head turned slightly to the right and with the chin near the right clavicle. He continued to occupy this position most of the time for the next six months, turning on the back occasionally but preferring the former position. $\mathrm{He}$ was not able to get on, or remain on, the left side at any time. When first seen by the writer the temperature was $101.5 \mathrm{~F}$. and the pulse 120 . $\mathrm{He}$ was still coughing and expectorating the muco-purulent matter. The writer was told that a sample had been sent to a microscopist of Detroit, who had reported himself unable to find any bacilli of tuberculosis. Another specimen was then sent to Ann Arbor and a similar report received from the microscopist in the University Laboratory. The patient was in fair flesh and though he had been told by his former attendant that his prospect of getting well was small he was in good spirits and very hopeful. He was in full possession of all his faculties and functions except those mentioned above. There was no material difficulty of deglutition, but the mouth could not be widely opened, and the appetite was fair. On account of the extreme sensitiveness of the neck it was impossible to make a satisfactory examination. Distortion of the spines of the cervical vertebræ was suspected, and sought for, but could not be demonstrated with certainty. A moderate blow on the top of the head with the hand caused pain in the vicinity of the cervical vertebræ. Pressure over the spines was not as painful as pressure at the sides of the neck about opposite the bodies of those bones. Percussion over the lower portion of the right mammary region elicited diminished resonance, and auscultation revealed diminished vesicular murmur over the whole mammary region of that side. Within a month the cough and expectoration first diminished and then completely ceased and did not return. The temperature continued uniformly and invariably in the immediate neighborhood of $101 \mathrm{~F}$. for more than six months and the pulse continued for the same length of time between 80 and 120 , being generallyabove 100. The slight fluctuations of temperature that occurred were without assignable cause, so far as could be determined. Within the first fortnight of the writer's attendance he discovered on auscultation a distinct friction or crepitation sound over the whole lower part of the right chest. It continued for about a week and then permanently disappeared, and vesicular murmur increased but never reached the normal. As the percussion note was nearly flat in the infra-mammary and infra-scapular regions effusion was suspected 